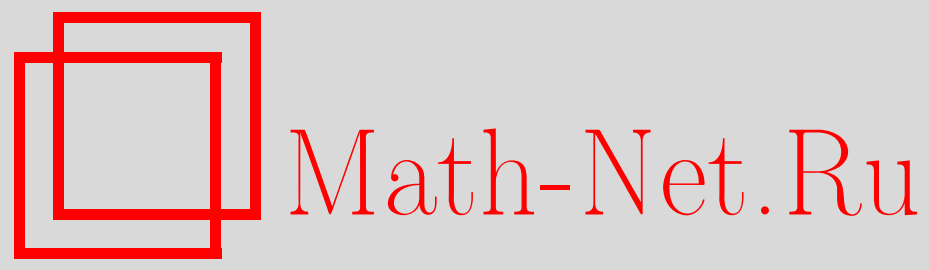

В. А. Треногин, Локально обратимые операторы и метод продолжения по параметру, Функи. анализ и его прил., 1996, том 30, выпуск 2, 93-95

DOI: https://doi.org/10.4213/faa530

Использование Общероссийского математического портала MathNet.Ru подразумевает, что вы прочитали и согласны с пользовательским соглашением

http://www . mathnet.ru/rus/agreement

Параметры загрузки:

IP: 35.173 .219 .149

26 апреля 2023 г., 11:18:53

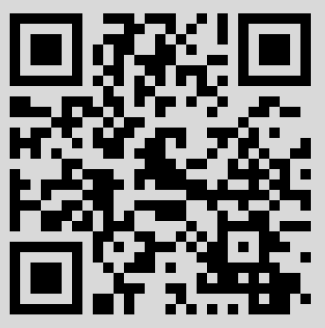




\title{
ЛИТЕРАТУРА
}

1. Альбеверио C., Гестези Ф., Хеэг-Крон P., Хольден Х. Решаемые модели в квантовой механике. Мир, М., 1991. 2. Gesztezy F., Kirsh W. J. Reine Angew. Math., 362, 28-50 (1985). 3. Михайлеи B. A. ДАН, 335, №4, 421-423 (1994). 4. Функциональный анализ (под ред. С. Г. Крейна). Наука, М., 1972. 5. Гехтман М. М., Станкевич И. В. Функц. анализ и его прил., 11, вып. 1, 61-62 (1977). 6. Christ C., Stolz G. J. Math. Anal. Appl., 184, No. 3, 491-516 (1994). 7. Simon B., Spencer Th. Commun. Math. Phys., 125, 113-125 (1989). 8. Шилов Г. Е., Фань Дьљ Тинь. Интеграл, мера и производная на линейных пространствах. Наука, М., 1967. 9. Кoчубей А. Н. Укр. матем. ж., 41, №10, 1391-1394 (1989). 10. Kamo T. Теория возмущений линейных операторов. Мир, М., 1972. 11. Бирман М. ШІ., Соломлк М. 3. Спектральная теория самосопряженных операторов в гильбертовом пространстве. Изд-во ЛГУ, Л-д, 1980.

Институт математики НАН Украины, Киев e-mail: mikhai@cksr.ac.bialystok.pl

Поступило в редакцию 25 февраля 1995 г.

УДК 517.988

\section{Локально обратимые операторы и метод продолжения по параметру}

\author{
(c) 1996. В. А. ТРЕНОГИн
}

Если для любых двух точек $x_{1}, x_{2}$ множества $\mathcal{D}$ задано неотрицательное число $\rho\left(x_{1}, x_{2}\right)$, удовлетворяющее аксиомам тождества и симметрии, то пару $(\mathcal{D}, \rho)$ назовем слабо метрическим пространством. При этом $\rho$ назовем слабой метрикой в $\mathcal{D}$. Все основные понятия теории метрических пространств переносятся на слабо метрическое пространство. Пусть $(\mathcal{D}, \rho)$ - слабо метрическое пространство, а $Y$ - нормированное пространство. Обозначим через $L_{\rho}(\mathcal{D}, Y)$ множество всех операторов $F: \mathcal{D} \rightarrow Y$, для которых конечна $\rho$-полунорма

$$
\|F\|_{\rho}=\sup _{x_{1}, x_{2} \in \mathcal{D}, x_{1} \neq x_{2}} \frac{\left\|F x_{1}-F x_{2}\right\|}{\rho\left(x_{1}, x_{2}\right)} .
$$

С естественными операциями сложения операторов и умножения оператора на число $L_{\rho}(\mathcal{D}, Y)$ является линейным полунормированным пространством. При этом операторы из $L_{\rho}(\mathcal{D}, Y) \rho$-непрерывны и $\rho$-ограничены, т. е. непрерывны и ограничены в смысле полунормы (1). Для каждого оператора $G: \mathcal{D} \rightarrow Y$ и любой слабой метрики $\rho$ введем числовую характеристику

$$
\llbracket G \rrbracket_{\rho}=\inf _{x_{1}, x_{2} \in \mathcal{D}, x_{1} \neq x_{2}} \frac{\left\|G x_{1}-G x_{2}\right\|}{\rho\left(x_{1}, x_{2}\right)},
$$

которую назовем $\rho$-мерой коэрцитивности оператора $G$.

Отметим, что если $\llbracket G \rrbracket_{\rho}>0$ хотя бы для одной слабой метрики $\rho$, то $G$ является мономорфизмом на $\mathcal{D}$. Нетрудно также проверить, что если $Y$ - банахово пространство, а $(\mathcal{D}, \rho)$ - слабо метрическое и $\rho$-полное пространство, 
то из $\llbracket G \rrbracket_{\rho}>0, G \in L_{\rho}(\mathcal{D}, Y)$, следует, что множество значений $R(G)$ оператора $G$ замкнуто в $Y$. Непосредственно из (1) и (2) следует такое предложение:

Лемма 1. Для любых $G: \mathcal{D} \rightarrow Y$ и $F \in L_{\rho}(\mathcal{D}, Y)$ справедливо ослабленное неравенство треугольника:

$$
\llbracket G+F \rrbracket_{\rho} \leqslant \llbracket G \rrbracket_{\rho}+\|F\|_{\rho} .
$$

Пусть теперь и всюду ниже $Y$ - банахово пространство, $A: \mathcal{D} \rightarrow Y, \square A \rrbracket_{\rho}>0$ и шар $S_{0}=\overline{S_{r_{0}}\left(y_{0}\right)}$ лежит в $R(A)$. Из этих условий следует, что уравнение $A x=y$ для любых $y \in S_{0}$ имеет в $\mathcal{D}$ единственное решение. Ниже будет показано, что аналогичным свойством обладает каждый оператор $B$, достаточно близкий к $A$ в смысле полунормы (1) и, кроме того, близкий к $A$ по норме пространства $Y$ в точке $x_{0}=A^{-1} y_{0}$. Этот результат при $R(A)=Y$ восходит к Банаху [1], см. также [2, 3]. Методом продолжения по параметру [3-8] подобные свойства обратимости в ряде случаев будут установлены и для операторов, относительно далеких от $A$.

Teоpema 1. Пусть $B, A \in L_{\rho}(\mathcal{D}, Y) u\|B-A\|_{\rho}<\llbracket A \rrbracket_{\rho}$. Тогдa $\llbracket B \rrbracket_{\rho}>0$ $u S_{r_{1}}\left(y_{1}\right) \subset R(B)$, где $y_{1}=B x_{0}, r_{1}=(1-q) r_{0}, a q=\|B-A\|_{\rho} \llbracket A \rrbracket_{\rho}^{-1}$.

Доказательство следует из леммы 1 и принципа сжимающих отображений.

СлЕДСТвиЕ 1. Если в условиях теоремы 1 для некоторого $\theta \in[0,1)$ выполнено неравенство $\left\|B x_{0}-y_{0}\right\| \leqslant(1-q) \theta r_{0}$, то $S_{r}\left(y_{0}\right) \subset R(B)$, где $r=(1-q)(1-\theta) r_{0}$.

СлеДСтвиЕ 2. Пусть $\llbracket A \rrbracket_{\rho}>0$ u $R(A)=Y$. Если $B, A \in L_{\rho}(\mathcal{D}, Y)$, $\|B-A\|_{\rho}<\llbracket A \rrbracket_{\rho}$, mo $\square B \rrbracket_{\rho}>0$ u $R(B)=Y$.

Введем теперь оператор-функцию $A(t), t \in[0,1]$, и положим $A(0)=A$, $A(1)=B$. Назовем ее $\rho$-непрерывной на $[0,1]$, если она непрерывна на $[0,1]$ по полунорме в $L_{\rho}(\mathcal{D}, Y)$, т. е. $A(t) \in L_{\rho}(\mathcal{D}, Y)$ и $\left\|A(t)-A\left(t_{0}\right)\right\|_{\rho} \rightarrow 0$ при $t \rightarrow t_{0}$ для любых $t, t_{0} \in[0,1]$. Оператор-функцию $A(t)$ назовем $\rho$-коэрцитивной на $[0,1]$, если

$$
\inf _{[0,1]} \llbracket A(t) \rrbracket_{\rho}=\gamma>0 .
$$

Теорема 2. Пусть оператор-функиия $A(t)$ $\rho$-непрерьвнна и $\rho$-коэриитивна на $[0,1]$, и $A(t) x_{0}=y_{0}$ nри $x_{0}=A^{-1} y_{0}$ для всех $t \in[0,1]$. Tогда $\| B]_{\rho}>0$ и существует $r_{1}>0$, такое, ито $S_{r_{1}}\left(y_{0}\right) \subset R(B)$. Если $R(A)=Y$, то и $R(B)=Y$. В этом случае условие $A(t) x_{0}=y_{0}$ излишне.

Доказательство проводится по схеме [3, п. 14.3].

Оператор-функцию $A(t)$ назовем $\rho$-липшиц-непрерывной на $[0,1]$ с постоянной $\delta$, если

$$
\sup _{t_{1}, t_{2} \in[0,1], t_{1} \neq t_{2}} \frac{\left\|A\left(t_{1}\right)-A\left(t_{2}\right)\right\|_{\rho}}{\left|t_{1}-t_{2}\right|}=\delta .
$$

Абстрактную функцию $A(t) x_{0}$ назовем липшиц-непрерывной на $[0,1]$ с постоянной $\beta$, если

$$
\sup _{t_{1}, t_{2} \in[0,1], t_{1} \neq t_{2}} \frac{\left\|A\left(t_{1}\right) x_{0}-A\left(t_{2}\right) x_{0}\right\|}{\left|t_{1}-t_{2}\right|}=\beta .
$$


ТЕорема 3. Пусть оператор-функиия $A(t)$ на $[0,1] \rho$-коэриитивна с постоянной $\gamma$ и иия $A(t) x_{0}$ липшич-непрерьвна с постоянной $\beta$ на $[0,1]$. Пусть $\alpha=\delta \gamma^{-1}$. Тогда если для некоторого натурального $m$ выполнень неравенства

$$
m>\alpha, \quad \beta\left(1+\frac{m-1}{m} \alpha\right)<\left(1-\frac{\alpha}{m}\right)^{m}\left(1-\frac{1}{m}\right)^{m-1} r_{0}
$$

mо $S_{r_{l}}\left(y_{0}\right) \subset R(A(t))$ для любого $t \in\left(t_{l-1}, t_{l}\right]$, где

$$
t_{l}=l / m, \quad r_{l}=(1-\alpha / m)^{m}(1-1 / m)^{m} r_{0}, \quad l=1, \ldots, m .
$$

Доказательство основано на применении следствия 1 теоремы 1 последовательно к парам операторов $A(t), t \in\left(t_{l-1}, t_{l}\right]$, и $A\left(t_{l-1}\right), l=1, \ldots, m$. При этом используется оценка $\left\|A(t) x\left(t_{0}\right)\right\| \leqslant \beta\left(1+\alpha t_{0}\right)\left|t-t_{0}\right|$, справедливая для решения $x\left(t_{0}\right)$ уравнения $A\left(t_{0}\right) x=y_{0}$.

ТЕОРемА 4. Пусть выполнень условия теоремы 3, но вместо неравенства (5) выполнено неравенство $\beta<(\alpha+1)^{-1} e^{-(\alpha+1)} r_{0}$. Тогда утверждение теоремь 3 справедливо для любого $m>\alpha$.

ЗАмЕчАНИЯ. 1. В условиях теорем 3 и 4 для любого $t \in[0,1]$ при $r=$ $e^{-(\alpha+1)} r_{0}$ справедливо включение $\overline{S_{r}\left(y_{0}\right)} \subset R(A(t))$. Если $\beta=0$, то можно взять $r=e^{-\alpha} r_{0}$.

2. Если $A(t)=A+t(B-A)$, то условия липшиц-непрерывности выполнены. При этом $\delta=\|B-A\|_{\rho}, \beta=\left\|B x_{0}\right\|$. Здесь очевидна роль ограничений малости на $\beta$. Если, например, $R(A)$ ограничена в $Y$, а $B x=A x+c$, то при достаточно большой $\|c\|$ уравнение $A(t) x=y_{0}$ не будет иметь решений на всем $[0,1]$.

3. Пусть оператор $F(x, t)$ отображает $\mathcal{D} \times(\alpha, \beta)$ в банахово пространство $Y$ и $F\left(x_{0}, t_{0}\right)=y_{0}$. Введем оператор-функцию $A(t)$ по формуле $A(t) x=$ $F(x, t)$. Перефразировка вышеизложенного приводит к новым локальным и глобальным теоремам о неявных операторах, т. е. к теоремам о решении уравнения $F(x, t)=y$.

\section{ЛИТЕРАТУРА}

1. Banach S. Studia Math., 1, 223-240 (1929). 2. Donden A. Rev. Math. Pure Appl., 25, No. 10, 1459-1475 (1980). 3. Треногин B. А. Функциональный анализ. Наука, М., 1993 (первое изд., 1980). 4. Bernstein S. N. Math. Ann., 59, 20-76 (1904). 5. Schauder J. Math. Z., 38, 257-282 (1934). 6. Давиденко Д. Ф. Укр. матем. ж., 7, №1, 18-28 (1955). 7. Fonarev A. A. Abstr. Am. Math. Soc., 4, No. 1, 133 (1983). 8. Гапоненко Ю. Л. ЖКурнал выч. матем. и матем. физики, 26, №8, 1123-1131 (1986).

Московский институт

Поступило в редакцию стали и сплавов

7 февраля 1995 г. 